\title{
Effects of Listening of $1 / f$ Fluctuation Music to the Changes of Heart Rate Variability in Daily Living Environment
}

\author{
Satoshi Watanabe ${ }^{\mathrm{a}, *}$, Ryouichi Takaue ${ }^{\mathrm{b}}$, Fenghui Yaoc, Yuji Matsumotod, \\ Hiroyuki Tsukamoto ${ }^{\mathrm{e}}$, Naruki Shirahama ${ }^{\mathrm{f}}$, Naofumi Nakaya ${ }^{\mathrm{g}}$ and Yukio Mori ${ }^{\mathrm{h}}$ \\ anternational University of Health and Welfare, Odawara-shi, 250-8588, Japan \\ bUniversity of East Asia, Shimonoseki-shi, 751-0807, Japan \\ 'Tennessee State University, Nashville, TN 37209, USA \\ ${ }^{\mathrm{d}}$ Kindai University, Higashiosaka-shi, 577-8502, Japan \\ 'Shizuoka Sangyo University, Fujieda-shi, 426-8668, Japan \\ fNational Institute of Technology, Kitakyushu College, Kitakyushu-shi, 802-0985, Japan \\ 'Tsukuba International University, Tsuchiura-shi, 300-0051, Japan

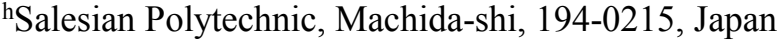 \\ *Corresponding Author: satoshi-w@iuhw.ac.jp
}

\begin{abstract}
In order to investigate the effects of listening of $1 / \mathrm{f}$ fluctuation music, analyses of heart rate variability (HRV) by discrete Fourier transform (DFT) have been widely conducted. These methods are useful to estimate the autonomic nervous system activity (sympathetic or parasympathetic activity). To examine the effects of listening of $1 / f$ fluctuation music, three experiments are carried out. 23 healthy subjects participated the first experiment, and one piece of $1 / \mathrm{f}$ fluctuation music is employed as the test piece. All subjects are asked to listen to the test piece individually, and their HRV are recorded and analyzed by DFT. The experiment result shows that the sympathetic nerve activity tends to decrease significantly when the subjects are listening to the test piece. The second invited 2 healthy subjects. They are asked to listen to the test piece (different from the first experiment) individually for 14 times (one time per a day), and their HRV are recorded and analyzed by DFT. The experiment result discovers linear relationship between the frequency of listening to the test piece and the changes of parasympathetic activity for both subjects. 5 healthy subjects participated the third experiment. They are asked to listen to the test piece (different from the first and second experiment) at the same time, and their HRV are recorded and analyzed by DFT. The experiment result shows that the sympathetic nerve activities of all subjects tend to decrease when they are listening to the test piece. From these results, this study
\end{abstract}

concludes: (1) the effect of decreasing in sympathetic nervous activity by listening of a $1 / \mathrm{f}$ fluctuation music is significant; (2) repeated listening of a 1 / f fluctuation music has effect to increase the parasympathetic activity.

Keywords: 1/f fluctuation music, heart rate variability, sympathetic nerve activity, parasympathetic nerve activity.

\section{Introduction}

Recently, many studies about effects of listening of music have been reported. For example, Watanabe et al investigated the fundamental study on effects of listening of wind band music to the changes of blood pressure and heart rate in daily living environment ${ }^{(1)}$. Furthermore, in order to investigate about effects of listening of music, analyses of heart rate variability (HRV) by discrete Fourier transform (DFT) also have been conducted broadly. These methods are useful to estimate the autonomic nerve activity such as sympathetic (index of tension or excitement) or parasympathetic (index of relaxation or sedation) activity. By using these methods, Hori et al studied the effects of music listening on $\mathrm{HRV}^{(2)}$, and so on. Therefore, the effects of the music listening for HRV have been, gradually, confirmed and accepted by many people.

However, factors of the effects of listening to music on HRV are not clear. Thus, $1 / \mathrm{f}$ fluctuation is considered as one of them ${ }^{(3)}$, effects of listening of $1 / \mathrm{f}$ fluctuation music to the 
changes of HRV are investigated.

In addition, listening to music is a quite common thing in daily life, it is also important that studies are carried out in daily living environment. However, some works largely have the implications of "experimental listening", and rarely study the effects of music listening in daily living environment $^{(1)(2)(4)}$. And some other works contain the various experiments in different specially designed environments ${ }^{(5)(6)}$. These conditions and setting may bring about mental and physical burden to the subjects, and the extra burden may affect the experiment result.

To examine the effects of listening of $1 / \mathrm{f}$ fluctuation music to the changes of HRV in daily living environment, three experiments are carried out. In the first experiment, 23 healthy subjects participated, and one piece of $1 /$ f fluctuation music is employed as the test piece. All subjects are asked to listen the test piece individually, and their HRV are recorded and analyzed by DFT. The second uses 2 healthy subjects. They are asked to listen to a test piece (different from the first experiment) individually for 14 times (one time per a day), and their HRV are recorded and analyzed by DFT. 5 healthy subjects participated in the third experiment. They are asked to listen to a test piece (different from the first and second experiment) at the same time, and their HRV are recorded and analyzed by DFT.

The remainder of this paper is organized as follows. Section 2 describes the theory of HRV analysis and fluctuations. Experiments are shown in Section 3. Results and discussion are given in Section 4. Section 5 shows the conclusions and future works.

\section{Theory of HRV Analysis and Fluctuations}

\section{$2.1 \quad$ HRV Analysis}

It can be regarded as synonymous with the pulse wave and heart rate analysis in healthy subjects ${ }^{(7)}$. The pulse wave can be measured easier than the heart rate. Therefore, it is possible that HRV analysis is carried out by the pulse wave analysis. In this paper, the pulse wave analysis is employed as an analysis method instead of HRV analysis.

The peak of the pulse wave obtained from the photoelectric pulse wave sensor attached to an earlobe or a finger of the subject. The pulse wave intervals (PI) are obtained from the peak of the pulse wave (see Fig.1).

In order to proceed a pulse wave analysis from the obtained PI, it is necessary to convert them to time series data (that is, the obtained PI will be interpolated to time series

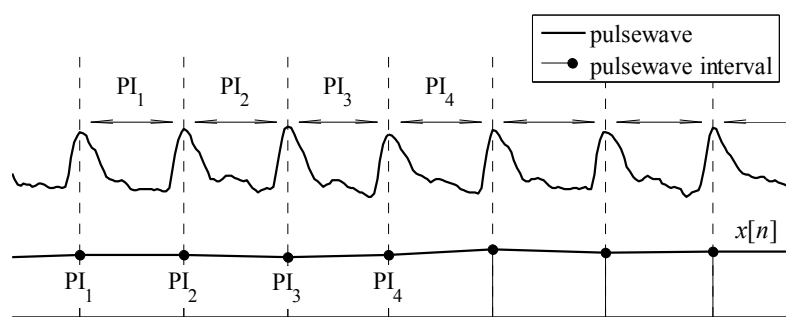

Fig. 1. Pulse wave and Pulse wave interval

data), because they are not a state of complete time series (not the isochronous intervals).

After the conversion step, the PI data which is in the time series is analyzed by the DFT. At the first step, frequency spectrum is obtained by DFT as shown in Eq. (1), the following components are extracted from them ${ }^{(2)}$.

LF (Low Frequency) components: $0.04 \mathrm{~Hz}-0.15 \mathrm{~Hz}$

$\mathrm{HF}$ (High Frequency) components: $0.15 \mathrm{~Hz}-0.4 \mathrm{~Hz}$

Subsequently, the periodogram is applied to estimate the power spectral density (PSD) of these regions (see Eq. (2) and (3)).

$$
X[k]=\sum_{n=0}^{N-1} x[n] e^{-j \frac{2 \pi k n}{N}},(k=0,1,2, \cdots, N-1)
$$

$$
L F=\frac{\sum\left|X_{L F}\right|^{2}}{f_{s} N}, \quad X_{L F}=\left\{X[k] \mid 0.04 \mathrm{~Hz} \leq \frac{k f_{s}}{N} \leq 0.15 \mathrm{~Hz}\right\}
$$

$$
H F=\frac{\sum\left|X_{H F}\right|^{2}}{f_{s} N}, \quad X_{H F}=\left\{X[k] \mid 0.15 \mathrm{~Hz} \leq \frac{k f_{s}}{N} \leq 0.40 \mathrm{~Hz}\right\}
$$

From the calculation result of Eq. (2) and (3), autonomic nervous activity (sympathetic or parasympathetic activity) indexes can be estimated as follows ${ }^{(2)}$.

$$
\begin{gathered}
\text { Sympathetic activity index }=\mathrm{LF} / \mathrm{HF} \\
\text { (the ratio of (2) to (3)) }
\end{gathered}
$$

$$
\begin{gathered}
\text { Parasympathetic activity index }=\mathrm{HF} \\
\text { (the value of (3)) }
\end{gathered}
$$

That is, the autonomic nervous activity is estimated by the value of Eq. (2) and (3).

According to the above preparation, the autonomic nervous activity by listening to the $1 /$ f fluctuation music can be estimated. 


\section{$2.2 \quad 1 / f$ fluctuation}

Fluctuation is defined as the deviation from the mean of a data. In the fluctuation, there are regular fluctuation (such as sound of the clock, predictable fluctuations) and irregular fluctuation (such as noise, unpredictable fluctuations). The fluctuations that exist between the regular fluctuation and irregular fluctuation are called $1 / \mathrm{f}$ fluctuation $^{(3)}$.

$1 / \mathrm{f}$ fluctuation is quantified as follows. A wave with fluctuation (such as music) is known to be decomposed in the frequency spectrum. In general, the spectral intensity $P$ which is inversely proportional to the frequency $f$ can be obtained according to Eq. (6) (where $\lambda$ is a constant).

$$
P=\frac{1}{f^{\lambda}}
$$

It is difficult to find $\lambda$ by directly solving Eq.(6). However, by taking the logarithm of Eq. (6), Eq. (7) is obtained, from which the value of $\lambda$ can be obtained easily.

$$
\log P=-\lambda \log f
$$

That is, the value of $\lambda$ is the value of the slope on the logarithmic plot on $P$ vs. $f$.

The fluctuations of the wave are summarized in Table 1 . Note that they are determined by the value of $\lambda$, if $\lambda=1$ then the wave has a $1 /$ f fluctuation. However, if $\lambda \fallingdotseq 1$ then it is can be call that the wave has a $1 / \mathrm{f}$ fluctuation actually.

\section{Experiments}

\subsection{Test Pieces (1/f fluctuation Music)}

Table 2 shows test pieces for the experiments. All of them have been recorded on commercial CD. Their fluctuations are examined as follows. First, the test pieces (see Table 2) are loaded into the personal computer (OS: windows) for analysis. Next, their instantaneous frequencies are obtained by zero-crossing method. Then power spectrum intensities $P$ are determined using them. Finally, logarithmic plots of $P v s$. frequencies $f$ are expressed. These analyzes are conducted by the software tool -Yuragi-Analyzer ${ }^{(8)}$. The results are summarized in Fig. 2 and 3.

The slope value, $\lambda$, is determined from the plot. Table 3 shows these results. From Table 3 , it is found that $\lambda$ value for all test pieces is close to 1.0. That is, it can be considered that all test pieces are $1 / \mathrm{f}$ fluctuation music.
Table 1. Fluctuations of the wave

\begin{tabular}{|c|c|}
\hline$\lambda$ & Fluctuations \\
\hline 0.0 & Irregular Fluctuations \\
\hline Close to 1.0 & $\boldsymbol{1} / \boldsymbol{f}$ Fluctuation \\
\hline 2.0 & Regular Fluctuations \\
\hline
\end{tabular}

Table 2. Test Pieces (1/f fluctuation Music)

\begin{tabular}{|c|c|}
\hline No.* & Test Piece's Name \\
\hline$(1)$ & "Requiem No.7 Lacrimosa" (Mozart) \\
\hline$(2)$ & "Appalachian Overture" (James Barnes) \\
\hline$(3)$ & "Sports Show March" (Yuji Koseki) \\
\hline \multicolumn{2}{|c|}{$*$ No. is indicated experiment No. }
\end{tabular}

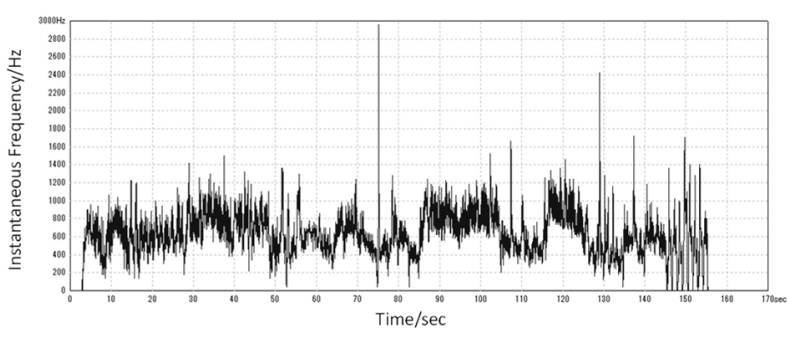

(1) "Requiem No.7 Lacrimosa" (Mozart)

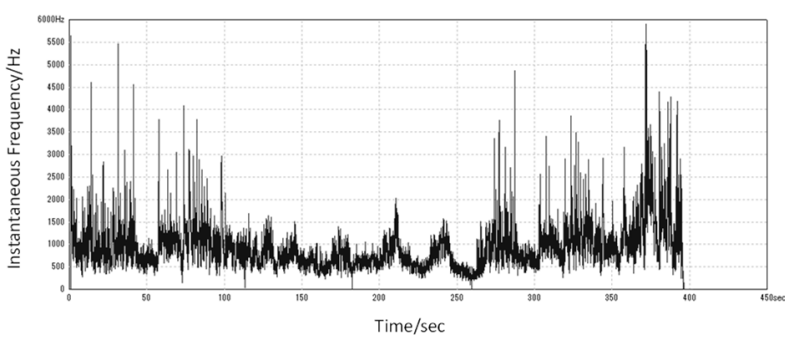

(2) “Appalachian Overture" (James Barnes)

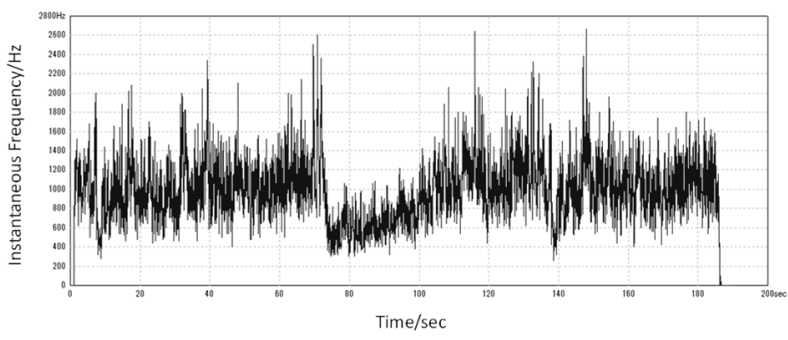

(3) “Sports Show March" (Yuji Koseki)

Fig. 2. Instantaneous Frequencies of Test Pieces 


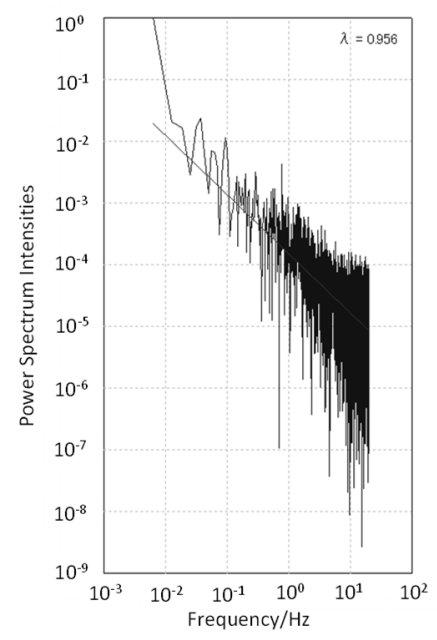

(1) "Requiem No.7 Lacrimosa" (Mozart)

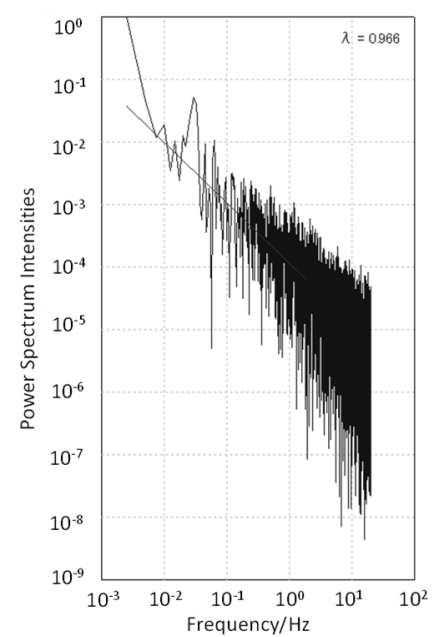

(2) “Appalachian Overture" (James Barnes)

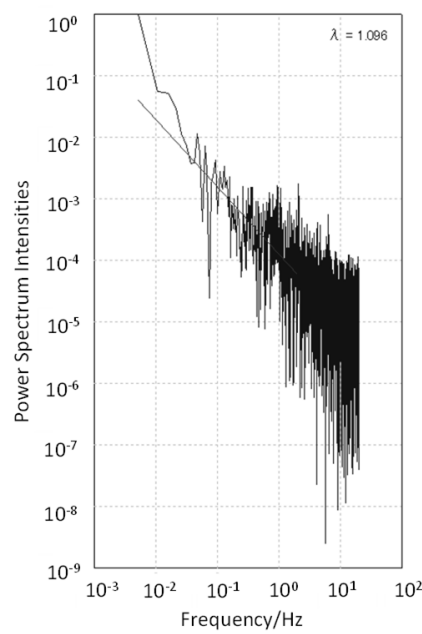

(3) "Sports Show March" (Yuji Koseki)

Fig. 3. Logarithmic Plots of $P$ vs. $f$

\subsection{Subjects}

Three experiments are carried out in this research. The subjects involved in these experiments are summarized in Table 4.

Ethical considerations are as follows:

The explanation of this study was given by the oral and documents to the subjects, and it was also given that participation in this study was voluntary. Furthermore, the data have been collected anonymously, and have been only used carefully for the presentation of this study.

\subsection{Experiment Environment}

The experiment is conducted in the daily living environment such as office, classroom and living room. The experiment employs the headphone (earphone) and a photoelectric pulse wave sensor attached to an earlobe or a finger of the subject. The subjects are requested to sit and do the experiment in closed-eye condition. No other special instruments is employed.

\subsection{Procedures of Experiments}

\subsubsection{Experiment (1)}

One piece of $1 / \mathrm{f}$ fluctuation music (see Table 2) is employed as the test piece. To play the test pieces, personal computer (OS: windows) is employed. 23 subjects are asked to listen to the test piece individually.

The flow of the experiment (1) is shown in Fig. 4 (1).

Table 3. $\lambda$ value (Determination of the fluctuation)

\begin{tabular}{|c|c|c|}
\hline No. & $\lambda$ value & Fluctuations \\
\hline$(1)$ & 0.956 & $1 /$ f Fluctuation \\
\hline$(2)$ & 0.966 & $1 /$ f Fluctuation \\
\hline$(3)$ & 1.096 & $1 /$ f Fluctuation \\
\hline
\end{tabular}

Table 4. Subjects for Experiments

\begin{tabular}{|c|c|}
\hline No. & Subjects \\
\hline$(1)$ & $23(15$ Males and 7 Females $)$ \\
\hline$(2)$ & 2 (Male and Female) \\
\hline$(3)$ & $5 \quad(2$ Males and 3 Females) \\
\hline
\end{tabular}


The peak of the pulse wave obtained from the photoelectric pulse wave sensor (U-MEDICA PDU-M100) attached to finger of the subject. Then, their HRV are recorded and analyzed by software (U-MEDICA “ARTETT”).

\subsubsection{Experiment (2)}

Two Subjects (one male and one female) are asked to listen to the test piece (see Table 2) individually for one time per a day (total: 14times). A commercial CD player (SONY D-120) is employed to play the test pieces. The flow of the experiment (2) is shown in Fig. 4 (2).

The peak of the pulse wave obtained from the photoelectric pulse wave sensor (SHIMADZU-RIKA CI6543) attached to earlobe of the subject. And their HRV are recorded and analyzed by software ("DATAStudio" (SHIMADZU-RIKA), "TSMaster" (S-TECH) and Microsoft Excel $\left.{ }^{\circledR}\right)$.

\subsubsection{Experiment (3)}

Five Subjects are asked to listen to the test piece (see Table 2) at the same time as shown in Fig. 5. A commercial CD player (SONY D-120) is employed to play the test pieces. The flow of the experiment (3) is shown in Fig. 4 (3).

The peak of the pulse wave obtained from the photoelectric pulse wave sensor (SHIMADZU-RIKA CI6543) attached to earlobe of the subject. And their HRV are recorded and analyzed by software ("DATAStudio" (SHIMADZU-RIKA), "TSMaster" (S-TECH) and Microsoft Excel $\mathbb{R})$.

\section{Results and Discussion}

\subsection{Experiment (1)}

All subjects' LF / HF indexes have been obtained from experiment (1). They are divided into three stage: before (before listening to the test piece), stimulation (listening to test piece), and after (after listening to the test piece), and their means and standard errors (SE) are calculated.

Fig. 6 shows results of these processes. When the subjects are listening to the test piece, it can be seen that the $\mathrm{LF} / \mathrm{HF}$ indexes are decrease significantly (t-test, $p<0.01$ ). It can be also seen that SE is smaller at that time. This fact is the evidence that the distribution of the LF / HF index is smaller than other stage, before and after.

From these results, it can say that sympathetic activity is decreased significantly when the subjects are listening to $1 /$ fluctuation music.

\subsection{Experiment (2)}

In experiment (2), 2 subjects (one male and one female) are asked to listen to the test piece for 14 times (one tine per day). In other words, 14 indexes have been obtained in this experiment for each subjects. Therefore, HF indexes at the

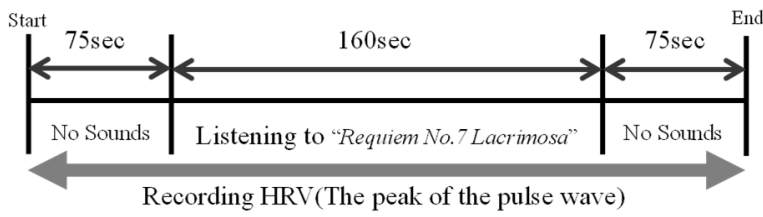

(1) Experiment (1)

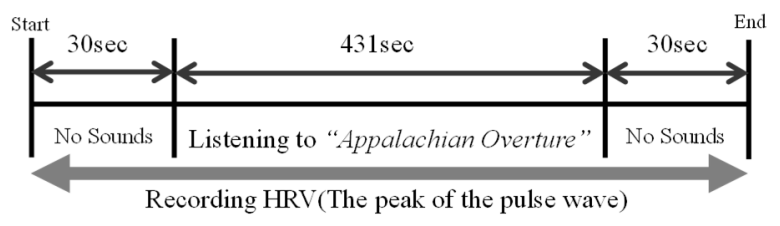

(2) Experiment (2)**

**This flow is repeated for 14 times (one time per a day)

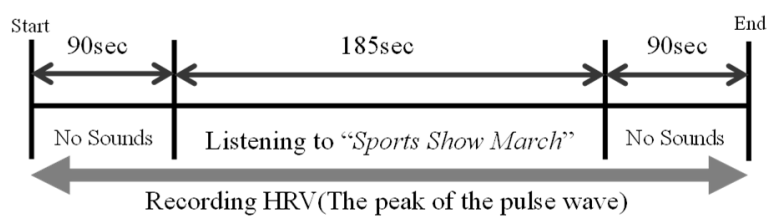

(3) Experiment (3)

Fig. 4. The flow of the experiment

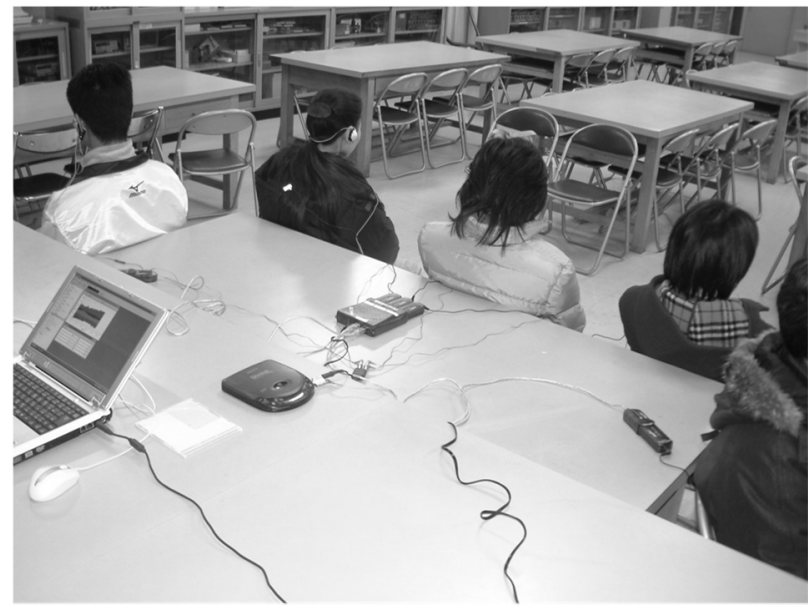

Fig. 5. The Photograph of Experiment (3) 
subjects are listening to the test piece in this experiment are noted, and they are plotted against listening times. These results are summarized in Fig. 7. It can be seen that a linear relationship exists between the frequency of listening to the test piece and the changes of HF indexes for both subjects.

This fact shows that parasympathetic activity is increased when the subjects are repeated listening to $1 / \mathrm{f}$ fluctuation music.

\subsection{Experiment (3)}

Experiment (3) is carried out under the conditions of simultaneous listening to the test piece as shown in Fig. 5. 5 subjects' LF / HF indexes have been obtained in this experiment. They are summarized in Fig. 8 as same as Fig. 6 . When the subjects are listening test piece, it is shown that all LF / HF indexes are decreased. However, it is necessary to consider whether this effect is caused by the simultaneous listening to the test piece or not.

From this fact and Section 4.1's results, it can say again that sympathetic activity is decreased significantly when the subjects are listening to $1 / \mathrm{f}$ fluctuation music.

\section{Conclusions}

To examine the effects of listening of $1 / \mathrm{f}$ fluctuation music, three experiments are carried out.

In the first experiment, 23 subjects are asked to listen to the test piece individually. The experiment result shows that the sympathetic nerve activity decreased significantly when the subjects are listening to $1 /$ f fluctuation music.

The second experiment employed 2 subjects. They are asked to listen to the test piece individually for 14 times. The experiment result discovers that a linear relationship exists between the frequency of listening to the test piece and the changes of parasympathetic activity for both subjects.

Five subjects participated in the third experiment. They are asked to listen to the test piece at the same time. The experiment result shows that the sympathetic nerve activities of all subjects decreased when they are listening to $1 / \mathrm{f}$ fluctuation music.

From these results, this study concludes two suggestions: (1) listening of a 1 / f fluctuation music has a significant effect of decreasing sympathetic nervous activity; (2) repeatedly listening of a 1 / f fluctuation music has an effect of increasing parasympathetic activity.

However, it is necessary to consider whether these two suggestions are caused by the conditions of above experiments or not. And in order to obtain reliable

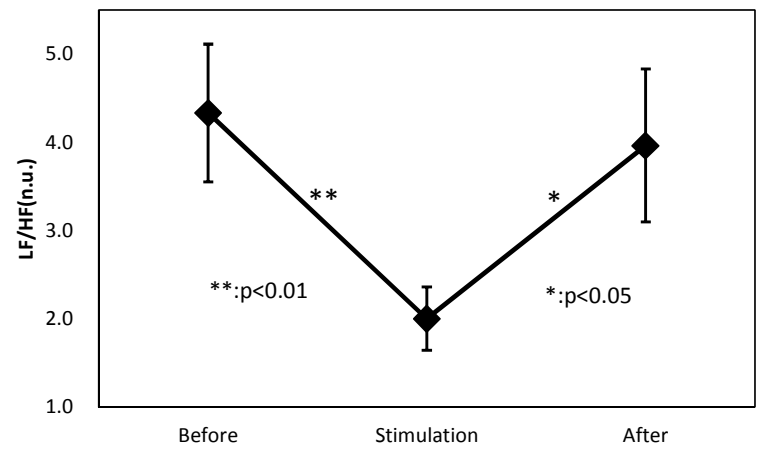

Fig. 6. Changes of LF/HF index in Experiment (1) $\checkmark$ : mean \pm standard error

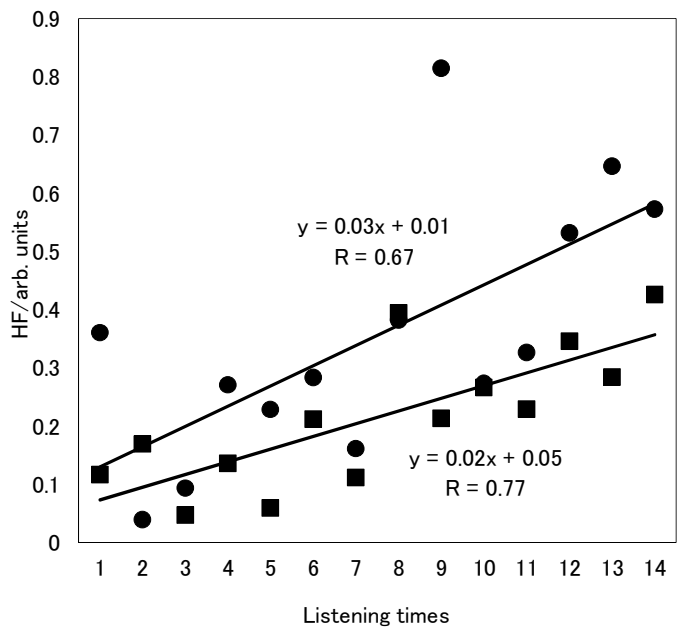

Fig. 7. Changes of HF indexes in Experiment (2) : subject A, $\mathbf{\square}$ : subject B

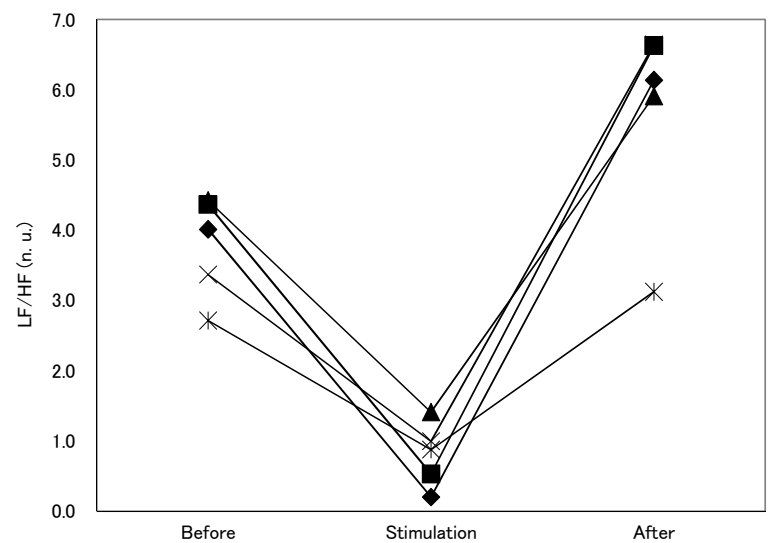

Fig. 8. Changes of LF/HF indexes in Experiment (3) 
conclusions, applying this method in the future study and conducting more investigation are very important.

Future works are as follows: (1) essence of the effects of listening of music, (2) variation of music, (3) essence of the effects of $1 / \mathrm{f}$ fluctuation, (4) classifying the subjects by their preference.

\section{Acknowledgment}

This work is partially supported by JSPS Grants-in-Aid for Scientific Research, No.16K01890.

\section{References}

(1) Satoshi Watanabe, Fenghui Yao and Ryouichi Takaue: "Fundamental Study on Effects of Listening of Wind Band Music to the Changes of Blood Pressure and Heart Rate in Daily Living Environment", Biomedical Soft Computing and Human Sciences, Vol. 12, No.1, pp.9-15, 2007

(2) Kiyokazu Hori, Yasutoshi Senga, Satoshi Minami and Seiki Hori: "Effects of listening to music on heart rate variability", Japanese Journal of Biometeorology, Vol. 41, No. 4, pp.131-140, 2004

(3) Toshimitsu Musha: "1/f fluctuation in biological systems", Proceedings 6th International Conference Noise in Physical System, pp. 143-146, 1981

(4) Benade, A. H.: "From instrument to ear in a room: Direct or via recording", Journal of Audio Engineering Society, Vol.33, No.4, pp.218-233, 1985

(5) Byers, J. F., Smyth, K. A.: "Effect of music intervention on noise annoyance, heart rate, and blood pressure in cardiac surgery patients", American Journal of Critical Care, Vol. 6, No.3, pp. 183-191, 1997

(6) Gardner, W. J., Lickliber J. C. R.: "Auditory analgesia in dental operations", Journal of the American Dental Association, Vol. 59, pp.1144-1149, 1959

(7) Toshiki Kikuchi, Yuji Sano, Jyunichi Abo, Shinsuke Urushidani and Tomoe Yamazaki: "Evaluation of Autonomic Nervous Activity using the Second Derivative Photoplothysmograph a-a Interval”, Journal of Sport Sciences and Osteopathic Therapy, Vol. 10, No. 3, pp.177-183, 2009 (in Japanese)

(8) "Yuragi-Analyzer": http://mahoroba.logicalarts.jp/category/software/yuragi-analyzer, as of 11 July 2017 (in Japanese) 